\title{
Surface-Bound Vascular Endothelial Growth Factor Promotes Prolonged Activation of Endothelial Cells: A New Technology for Capturing Endothelial Progenitor Cells by Intravascular Stents
}

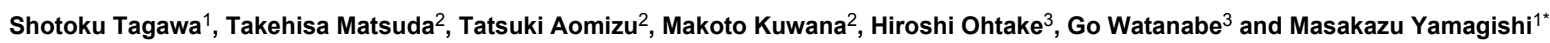 \\ ${ }^{1}$ Division of Cardiovascular Medicine, Kanazawa University Graduate School of Medicine, Kanazawa, Japan \\ ${ }^{2}$ Genome Biotechnology Laboratory, Kanazawa Institute of Technology, Japan \\ ${ }^{3}$ Division of Cardiovascular Surgery, Kanazawa University Graduate School of Medicine, Ishikawa, Japan \\ *Corresponding author: Masakazu Yamagishi, MD, PhD, Division of Cardiovascular Medicine, Kanazawa University Graduate School of Medicine, 13-1 Takara-machi, \\ Kanazawa, 920-8641 Ishikawa, Japan, Tel: 81-76-265-2259; Fax: 81-76-234-4210; E-mail: myamagi@med.kanazawa-u.ac.jp
}

Received date: April 10, 2014; Accepted date: July 11 2014; Published date: July 14, 2014

Copyright: (C) 2014 Tagawa S, et al. This is an open-access article distributed under the terms of the Creative Commons Attribution License, which permits unrestricted use, distribution, and reproduction in any medium, provided the original author and source are credited.

\begin{abstract}
In -situ selective capture of the Endothelial Progenitor Cell (EPC) in the arterial blood stream can provide antithrombogenic and -cell proliferation potential to implanted intravascular stents. Therefore, we defined molecular mechanisms of EPC activation associated with stent surface-bound proteins. We sought suitable bound protein to capture and proliferate EPCs. Then, to determine whether and how long the surface-bound protein activates intracellular signal-transduction pathways of endothelial cells through its receptor, we studied the phosphorylation of key intracellular macromolecules including Vascular Endothelial Growth Factor (VEGF) Receptor (R)-2 (VEGFR-2), focal adhesion protein-tyrosine kinase, Akt, and extracellular signal-regulated kinase in human umbilical vein endothelial cells. We found the most suitable surface-bound protein was VEGF. Phosphorylation of these macromolecules continued for a long time up to 72 hours. Under these conditions, quantitative RT-PCR revealed time-dependent up-regulation of the mRNAs encoding three major extracellular matrix macromolecules, collagen IV, laminin-5, and fibronectin. Immuno histo- chemical analysis revealed that these macromolecules were secreted on the basal sides of adherent cells over time and that within a few days after initial adhesion occurred, deposition of these macromolecules shut down the EC adhesion. These results demonstrate that activation of the VEGF-VEGFR intracellular signaling pathway is significant for in situ EPC capture technology for intravascular stents, although further in vivo studies should be done to confirm these processes.
\end{abstract}

Keywords: Surface-bound VEGF; Endothelial cell; Intracellular signal-transduction pathway; Adhesion mechanism

\section{Introduction}

Although synthetic grafts of small diameter $(<5 \mathrm{~mm})$ have yielded very limited success in coronary, cerebral, and peripheral vascular repairs significant progresses have been reported with coronary stents. However, further improvement of anti-thrombogenic and -cell proliferative potential is necessary $[1,2]$. In animal models and clinical studies, ex vivo seeding of autologous Endothelial Cells (ECs) harvested from veins, or Endothelial Progenitor Cells (EPCs) collected from peripheral blood, onto stents has yielded significant positive outcomes leading to very high patency without appreciable thrombus formation, intimal hyperplasia, narrowing, or occlusion [3-7]. However, we and others have shown that cell therapies using such ex vivo seeding techniques have not been used routinely in the clinic due to the tedious fabrication process [5-8]. To circumvent these drawbacks, technologies designed to capture cells in situ in the blood stream have been proposed based on several bioactive platforms [9-15]. One such platform makes use of anti-CD34 antibody bound to the surface of stents $[11,12,14]$. Since CD34 is expressed on endothelial lineage cells as well as other hematopoietic cells, anti-CD34 antibodybound stents capture EPCs but blood cell such as macrophages also adhere onto the stent surfaces, resulting in the formation of heterogeneous, immature endothelial-like tissues.
Our strategy for achieving highly selective capture of EPCs has been to explore bio-molecular targeting of cell-membrane proteins exclusively expressed on endothelial-lineage cells. We previously reported that surface-bound Vascular Endothelial Growth Factor (VEGF) serves as an adhesion platform in vitro. Following adhesion to VEGF-bound surfaces, human EPCs undergo differentiation to mature ECs [16-19]. In addition, the tight binding of human ECs and EPCs, both of which adhere to VEGF-bound surfaces, enable them to withstand simulated arterial shear stress [17].

Recently, we found that VEGF-coated stents actually capture circulating EPCs at a very early period of stenting in a porcine coronary model, followed by rapid full endothelialization [19]. In the present study, we determined (1) which protein could be best suited for coating of stents to capture endothelial-lineage cells, (2) whether and how long surface-bound protein activates four key molecules involved in intracellular signal-transduction pathways such as VEGFR, FAK, Extracellular Signal-Regulated Kinase (ERK), and Akt and (3) how long the adhesion mediated by binding of the protein to its receptor lasts.

\section{Methods}

\section{Protein-bound substrate}

Protein-bound glass substrates were prepared via a sequential fourstep procedure, as reported previously [18]: (1) coating of 
Citation: Tagawa S, Matsuda T, Aomizu T, Kuwana M, Ohtake H, et al. (2014) Surface-Bound Vascular Endothelial Growth Factor Promotes Prolonged Activation of Endothelial Cells: A New Technology for Capturing Endothelial Progenitor Cells by Intravascular Stents. J Tissue Sci Eng 5: 1000140. doi:10.4172/2157-7552.1000140

Page 2 of 7

poly(ethylene-co-vinyl alcohol) (PEVA; 68 mol\% of vinyl alcohol unit, purchased from Poly-Science, Cleveland, OH, USA) dissolved in Hexafluoroisopropyl alcohol (HFIP, Central Glass Chemicals, Tokyo, Japan) on a custom-made round glass substrate (Ma tsunami Glass, Osaka, Japan), using a spin coater. The thickness of the coated layer was approximately $5 \mu \mathrm{m}$. (2) Activation of surface hydroxyl groups of PEVA with N, N'-carbodiimidazole (Wako Pure Chemicals, Osaka, Japan) in acetonitrile solution. (3) Chemical conjugation of protein to the surface via activated surface hydroxyl groups. (4) Deactivation or blocking of excess activated surface hydroxyl groups with 2(aminoethoxy) ethanol (Tokyo Kasei Co., Tokyo, Japan).

Proteins used for binding to the glass substrates were as follows: recombinant VEGF165, referred to as VEGF (PeproTech, Rocky Hill, NJ, USA), anti-human VEGFR-1 and anti-human VEGFR-2 (R\&D Systems Minneapolis, MN, USA), anti-Tie1 antibody (T2817), antiTie2 antibody (T3192), and fibronectin (FN, F4759) (Sigma, Chicago, IL, USA). High-density binding of proteins to the surface was confirmed by fluorescence-spectroscopic analysis using a fluorescently labeled protein, also as previously reported [18].

\section{Cell Culture}

In the present study, human ECs were used rather than EPCs, as a cell type representative of endothelial-lineage cells, because once EPCs are isolated from blood, they differentiate into mature ECs over the course of culturing, and the nature and phenotype of EPCs vary with time. Human umbilical vein ECs (Bio-Science, Cambrex, CA, USA) were seeded onto protein-bound glass substrates placed in each well of 96-well culture plates (Corning, Corning, NY, USA) at $2.5 \times 104$ cells/ well and cultured in complete EBM-2 culture medium supplemented with EGM-2MV medium (please give percent of supplement) (Lonza, Basel, Switzerland) at $37^{\circ} \mathrm{C}$ in $5 \% \mathrm{CO} 2$, according to a commonly used method [5-7]. One day before the investigation of intracellular signaltransduction pathways, cells were cultured in serum- and growth factor-free EGM-2 medium for 24 hours, and then phosphorylation of proteins was determined.

Phosphorylated ERK was measured using the CASETM Kit for ERK1/2 (FE002, SA Biosciences, Frederick, MD, USA) by an ELISA technique according to the manufacturer's protocol. The assay results were determined using a micro-plate reader (Model 680, Bio-Rad, Tokyo, Japan). The effect of inhibition of ERK signaling was investigated by addition of an MEK1/2 inhibitor, U0126 (9903, Cell Signaling Technology, Tokyo, Japan) to EBM-2 culture medium at a dose of $20 \mu \mathrm{l} / \mathrm{ml}$ during the serum- and growth factor-free culturing period.

\section{Phosphorylation of VEGFR-2, FAK, and Akt}

ECs detached from substrates were lysed at $4{ }^{\circ} \mathrm{C}$ in lysis buffer $(10$ $\mathrm{mM}$ Tris- $\mathrm{HCl}$ [pH 7.6], $150 \mathrm{mM} \mathrm{NaCl}, 30 \mathrm{mM} \mathrm{Na} 4 \mathrm{P} 2 \mathrm{O} 7,50 \mathrm{mM} \mathrm{NaF}$, $2 \mathrm{mM} \mathrm{Na} 3 \mathrm{Vo} 4,1 \mathrm{mM}$ EDTA, $1 \mathrm{mM}$ phenylmethylsulfonyl fluoride) at $4^{\circ} \mathrm{C}$. The cell lysates were centrifuged at $13,600 \times \mathrm{g}$ and the supernatants were collected and the protein concentration of the supernatant was determined by the Bradford method using the Protein Assay Dye Reagent Concentrates (Bio-Rad, Tokyo, Japan). The supernatants $(5 \mu \mathrm{g}$ protein each) were subjected to Sodium Dodecyl Sulfate Polyacrylamide Gel Electrophoresis (SDS-PAGE; 7.5\% polyacrylamide gel) to separate the proteins. After electrophoretic transfer of separated proteins to a poly (vinylidene difluoride) membrane (Immobilon, Millipore, Bedford, MA, USA) for 2 hours at
$100 \mathrm{~V}$, blots were probed with primary antibodies (dilution: $1 / 1,000$ ), followed by treatment with horseradish peroxidase-conjugated secondary antibody, either anti-rabbit IgG $(\mathrm{H}+\mathrm{L})$ (Invitrogen, Tokyo, Japan) or anti-mouse IgG linked whole antibody (NA931V, GE Healthcare, Tokyo, Japan) (dilution; 1/1,000).

Immuno-blots were visualized using Super Signal West Pico Chemiluminescent Substrate (Thermo Fisher Scientific, Waltham, MA, USA) on a Lumino-Image analyzer (LAS-3000, FUJIFILM, Tokyo, Japan). Densito-metric quantification was carried out using the Image J software (NIH, USA). Primary antibodies against nonphosphorylated and phosphorylated proteins were as follows: nonphosphorylated VEGF-R2, anti-VEGF receptor 2 (55B11) rabbit mAb (Cell Signaling Technology, Inc., Danvers, MA, USA); phosphorylated VEGFR-2 (pVEGFR-2), anti-phospho-VEGFR-2 (Tyr1175) (D5B11) rabbit $\mathrm{mAb}$ (Cell Signaling Technology); FAK, mouse anti-human FAK antibody (610088) (BD Biosciences, Sparks, MD, USA); p-FAK; mouse anti-human p-FAK antibody (Tyr397) (BD Biosciences); Akt, anti-pan-Akt (C67E7) rabbit mAb (\#4691, Cell Signaling Technologies); p-Akt, anti-phospho-Akt (Ser473) (193H12) rabbit mAb (\#4058, Cell Signaling Technologies). VEGF binds to two structurally related tyrosine kinase receptors (VEGFRs) denoted VEGFR-1 (also known as Flt-1) and VEGFR-2 (also known as KDR). Because the binding and auto-phosphorylation of KDR are much more efficient than those of Flt-1, KDR (VEGFR-2) was selected for this study.

\section{Quantitative RT-PCR}

m-RNA was extracted from ECs that were cultured in serum and growth factor-free medium for one day before measurement. m-RNA quantitation according to the cell-culture protocol described above, using RN easy Micro kit (QIAGEN, Tokyo, Japan). RNA concentration was determined by spectrophotometry. c-DNAs were synthesized using the High Capacity RNA-to-c-DNA kit (Applied Biosystems, Osaka, Japan) and a T-Gradient DNA thermal cycler (Biometra, Gettingen, Germany).

Quantitative real-time Reverse-Transcription Polymerase Chain Reaction (RT-PCR) was performed using the QuantiTect SYBR Green PCR Kit and detection on an Applied Bio-systems 7500 Real time PCR System (Applied Bio-systems, Tokyo, Japan). RT-PCR conditions were as follows: initial denaturation, $95^{\circ} \mathrm{C}$ for $10 \mathrm{~min}$, followed by 40 cycles of $95^{\circ} \mathrm{C}$ for 15 seconds and $60^{\circ} \mathrm{C}$ for 60 seconds. For each c-DNA sample, GAPDH (Glyceraldehyde 3-Phosphate Dehydrogenase) was also amplified for purpose of normalization, as described below.

The primer pairs used for amplifications were designed by Nihon Gene Laboratories, Inc. (Sendai, Japan); forward and reverse primer sequences are shown in Table 1. All PCR data were calculated using the $\Delta \Delta \mathrm{Ct}$ method [20]. For all samples, the data were normalized to the levels of the mRNA for the housekeeping gene GAPDH $(\Delta \mathrm{Ct} 1=$ $\mathrm{Ct} 1$ [gene of interest] - Ct1 [GAPDH]) and further normalized to the levels of GAPDH mRNA in the cells after 2 hours of culture $(\Delta \mathrm{Ct} 0$ [gene of interest] - Ct0[GAPDH]) under the same conditions. The data are therefore presented as the ratios of the expression levels of mRNAs of interest relative to their levels after 2 hours of culture. Each sample was analyzed in triplicate.

\begin{tabular}{|l|l|}
\hline Gene & Primer sequence \\
\hline GAPDH & \\
\hline
\end{tabular}


Citation: Tagawa S, Matsuda T, Aomizu T, Kuwana M, Ohtake H, et al. (2014) Surface-Bound Vascular Endothelial Growth Factor Promotes Prolonged Activation of Endothelial Cells: A New Technology for Capturing Endothelial Progenitor Cells by Intravascular Stents. J Tissue Sci Eng 5: 1000140. doi:10.4172/2157-7552.1000140

Page 3 of 7

\begin{tabular}{|c|l|}
\hline Forward & 5'ATGACATCAAGAATGGTG-3' \\
\hline Reverse & 5'-CTGTAGCCAAATTCGTTGTC-3' \\
\hline Collagen type IV & \\
\hline Forward & 5'-CTAATCACAAACTGAATGACTTGACTTCA-3' \\
\hline Reverse & 5'-AAATGGCCCGAATGTGCTTA-3' \\
\hline FN & \\
\hline Forward & 5'-TGGAC CAGAGATCTTGGATGTTC-3' \\
\hline Reverse & 5'-CGCCTAAA ACCATGTTCCTCA-3' \\
\hline Laminin-5 & \\
\hline Forward & 5'-GGCTCACTCTGTATTGTTGG-3' \\
\hline Reverse & 5'-ACAGAGACTGCTTTGGTGTG-3' \\
\hline
\end{tabular}

\section{Statistical Analysis}

Results are presented as means with standard errors. Differences in relative gene expression were analyzed using a two-way ANOVA test. A p-value of less than 0.05 was considered statistically significant.

\section{Results}

\section{Effect of surface-bound proteins on EC adhesion and proliferation}

On VEGF-bound substrate, at initial 2 hours after seeding and the time-dependent increase in cell density were almost identical to those on FN-bound substrate (Figure1 and 2). In contrast, much lower proliferative potentials were observed when anti-receptor antibodies were bound to the substrate. Morphologically, SEM images of cultured ECs on these protein-bound substrates revealed that cells on VEGFbound substrate, similar to those on FN-bound substrate

Table 1: Forward and reverse primers used for real-time PCR assay of ECM genes

\section{Immunohisto-chemical staining}

Cells adhering to the glass substrates were washed twice with Phosphate-Buffered Saline (PBS), and then immersed into 4\% formalin in PBS for 30 minutes at room temperature. Samples were rinsed 3 times with PBS, and then blocked with $1 \%$ Bovine Serum Albumin (BSA) in PBS for $60 \mathrm{~min}$ at room temperature. After the substrates were rinsed with PBS, the primary antibodies were added and incubated for 18 hours at $4^{\circ} \mathrm{C}$. Samples were next rinsed in PBS, and then incubated for 50 min with Alexa Fluor 488-conjugated antirabbit IgG. Samples were then rinsed 3 times for 5 minutes each in PBS. All antibodies were diluted with PBS containing $0.1 \%$ Triton and $1 \%$ BSA at used at a dilution of 1:100 for the primary antibody and $1: 150$ for the secondary antibody.

Negative controls using secondary antibodies alone were performed for each antibody. The fluorescently labeled secondary antibodies used were as follows: Fibronectin (FN), mouse monoclonal anti-fibronectin antibody (ab6328, Abcam, Tokyo, Japan); collagen type IV, rabbit polyclonal anti-collagen IV antibody (ab21295, Abcam); laminin-5, mouse monoclonal anti-laminin-5 antibody, clone P3E4 (MAB1949, Millipore, Tokyo, Japan); Alexa Fluor 594 goat anti-rabbit IgG (A-11012, Invitrogen); and Alexa Fluor 488 goat anti-mouse IgG (A-11001, Invitrogen). The cell nuclei were stained with Hoechst 3334 stain (H1399, Invitrogen). Images were recorded on a BZ-8000 fluorescence microscope (Keyence, Osaka, Japan) at $10 \times$ magnification.

\section{Scanning Electron Microscopy}

An S3400-NX Scanning Electron Microscope (SEM; Hitachi HighTechnologies Co., Tokyo, Japan) was used and operated at an acceleration voltage of $10 \mathrm{kV}$. The samples were freeze-dried and then sputter-coated with osmium (VIII) oxide using a Neoc-ST osmium coater (Meiwa Forces, Osaka, Japan).

Figure 1: Schematic presentation of possible interactions between surface-bound proteins and cell-membrane receptors. Cell-surface receptors include integrin, which interacts with adhesive proteins such as Fibronectin (FN), VEGF receptor (VEGFR-1 and VEGFR-2), and Tie-1 and Tie-2, all of which are specific to Endothelial Cells (ECs) and their progenitor cells (EPCs). Ligands are FN, VEGF, and corresponding anti-receptor antibodies

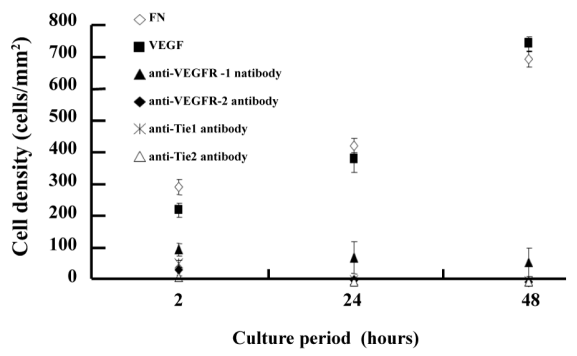

Figure 2: Culture period-dependent cell density of Endothelial Cells (ECs) on various protein-bound substrates. Note that EC density increased only on VEGF- and FN-bound substrate 
Citation: Tagawa S, Matsuda T, Aomizu T, Kuwana M, Ohtake H, et al. (2014) Surface-Bound Vascular Endothelial Growth Factor Promotes Prolonged Activation of Endothelial Cells: A New Technology for Capturing Endothelial Progenitor Cells by Intravascular Stents. J Tissue Sci Eng 5: 1000140. doi:10.4172/2157-7552.1000140

Page 4 of 7

FN-bound substrate
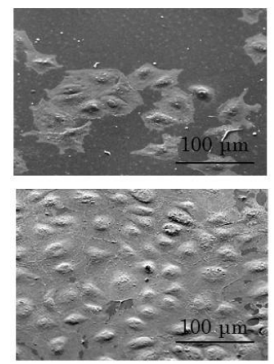

VEGF-bound substrate anti-VEGFR-2-bound substrate

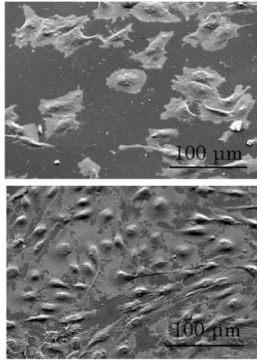

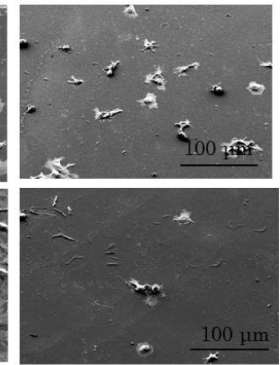

Figure 3: Representative scanning electron microscopic images of endothelial cells cultured on various ligand-bound substrates for 2 hours (upper) and 48 hours (lower). Cells adhere, spread, and proliferate well on FN- (left) and VEGF-bound (center) substrates but neither cell spreading nor proliferation was observed on antiVEGFR-2 receptor antibody-bound substrates (right). Scales are 100 micrometers

By contrast, cells on all the anti-receptor antibody-bound substrates exhibited at an early period of culture; and after longer culturing times, primarily fragmented cell debris were observed. Thus, among the proteins we examined, only surface-bound VEGF exhibited high cell-adhesion, spreading, and proliferation potentials and these properties were similar to those of FN-bound substrate.

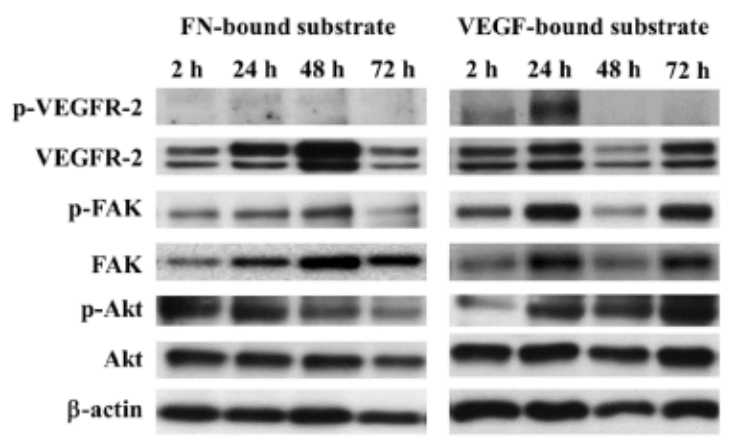

Figure 4: Western-blot analyses of phosphorylation of VEGFR-2, FAK, and Akt, by SDS-PAGE. Endothelial cells cultured on VEGFand FN-bound substrates were starved for 24 hours before the indicated times, and then lysed and supernatants were obtained. Equal amounts of protein were loaded in the lanes. Immunoblotting was performed with anti-phospho-VEGFR-2 antibody, anti-phospho-FAK antibody, and anti-phospho-Akt antibody as well as antibodies specific for the corresponding un-phosphorylated proteins. One day before the measurement of phosphorylation, culture medium was replaced with serum- and growth factor-free EBM-2 medium

\section{Receptor-mediated phosphorylation}

Figure 4 shows representative expression profiles of proteins and their phosphorylated forms on both VEGF- and FN-bound substrates.
From the Western blots, we quantitated the level of autophosphorylation at Tyr1175 of VEGFR-2, Tyr397 of FAK, and Ser473 residue of Akt. Figures 5A, B and Figure 6A show the ratios of phosphorylated protein to total protein for each of these proteins. For VEGF-bound substrate, the level of phosphorylation (p-VEGFR-2/ total VEGFR-2) increased in the early period of culture (24 hours), but little phosphorylation was observed at later times (Figure 5A).
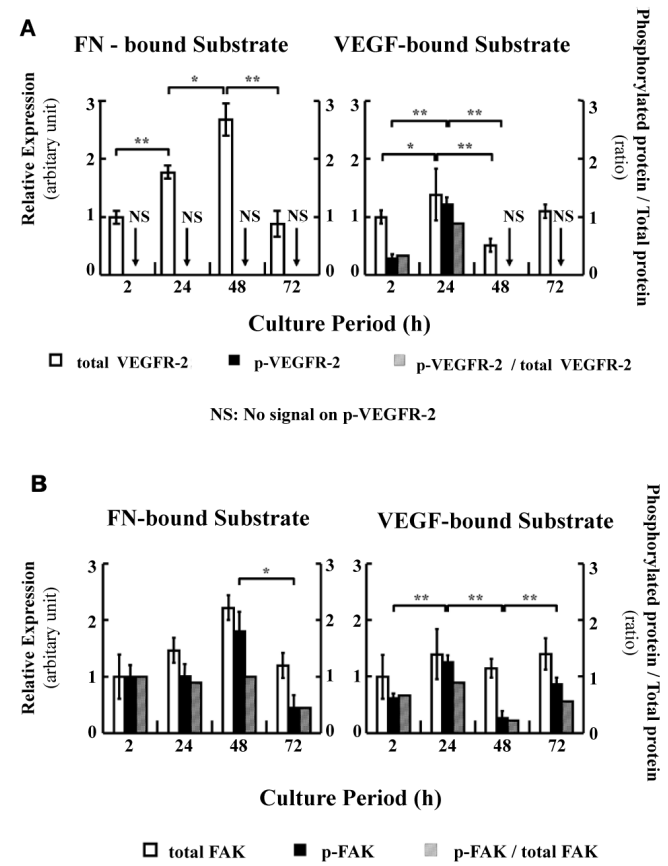

Figure 5: Western-blotting analysis of VEGFR-2 phosphorylation (upper) and FAK phosphorylation (lower). Relative activity was calculated from the amount of phosphorylated protein normalized to that of total protein, which was determined by densito-metric quantification using NIH Image J software $(\mathrm{n}=3)$. NS: no signal. Data are expressed as means \pm standard deviation for 3 independent samples. ${ }^{*} \mathrm{p}<0.05,{ }^{* *} \mathrm{p}<0.01$

On the other hand, little phosphorylation occurred on FN-bound substrate at any time during the culture period. On both VEGF-bound and FN-bound substrates, the ratio of phospho-FAK to total FAK increased in the early period of culture times, but tended to decrease after prolonged culture (Figure 5B). On VEGF-bound substrate, the ratio of phospho-Akt to total Akt increased continuously with culture time, whereas on FN-bound substrate the level of Akt phosphorylation tended to decrease over time (Figure 6A). ERK phosphorylation analysis determined by ELISA after culturing for various times (Figure $6 \mathrm{~B})$ revealed that elevated phosphorylation of ERK on VEGF-bound substrate continues for a period of 72 hours. Upon addition of U0126, a potent inhibitor of mitogen-activated protein kinase (MEK1/2), which is located upstream of ERK signaling pathway, ERK phosphorylation was reduced to control levels. In contrast, a timedependent increase in ERK phosphorylation was observed on FNbound substrate [21]. Thus, there was significant difference in ERK phosphorylation between VEGF and VEGF with U0126. 
Citation: Tagawa S, Matsuda T, Aomizu T, Kuwana M, Ohtake H, et al. (2014) Surface-Bound Vascular Endothelial Growth Factor Promotes Prolonged Activation of Endothelial Cells: A New Technology for Capturing Endothelial Progenitor Cells by Intravascular Stents. J Tissue Sci Eng 5: 1000140. doi:10.4172/2157-7552.1000140

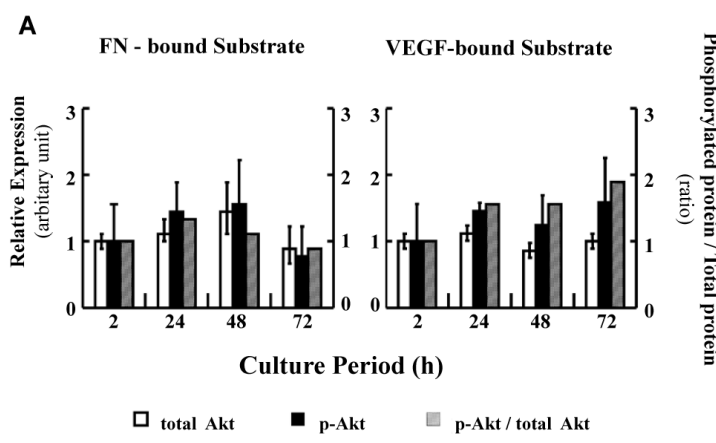

B

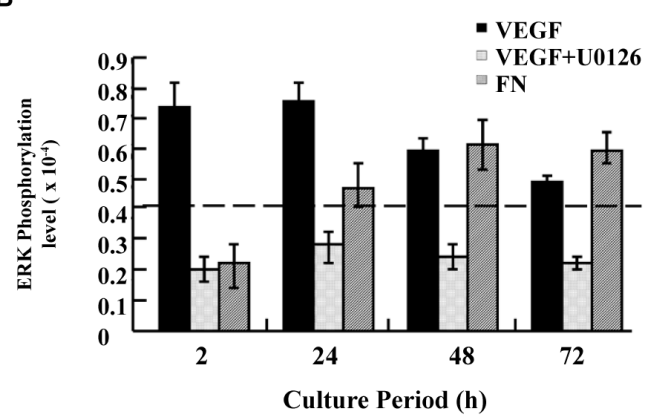

Figure 6: Phosphorylation profiles of Akt in FN-bound (upper left), and VEGF-bound (upper right), and ERK (lower) in cultured cells. For ELISA analysis, cells were starved for 24 hours before the indicated times, and then lysed $(\mathrm{n}=3)$. U0126, an inhibitor of MEK1/2 (upstream of ERK signal pathway), was added to the medium at a dose of $20 \mu \mathrm{g} / \mathrm{ml}$. (Note that a separate experiment showed that ERK activation level was at similar doses). The dotted line indicates the maximum inhibition of ERK activation via the MEK1/2 pathway. Data are expressed as means \pm standard deviation for 3 independent samples

\section{Expression of mRNAs encoding extracellular matrix (ECM) proteins}

When we monitored the relative change in the levels of these ECM m-RNAs as a function of culture time (Figure 7). On VEGF-bound substrate, both collagen IV and FN expression tended to increase with culture time. On FN-bound substrate, collagen expression increased slightly with culture time, whereas FN expression decreased. Laminin expression on both protein-bound substrates remained unchanged.

\section{ECM deposition by immunohistochemistry}

Qualitative observation of samples revealed that deposition of ECM molecules on the basal side of the adhered cells increased with culture time, regardless of the types of ECM molecules (Figure 8): there was little deposition of ECMs at 2 hours, but some deposition of all the ECM proteins was observed at 24 hours. At 72 hours, significant deposition of these proteins was observed.

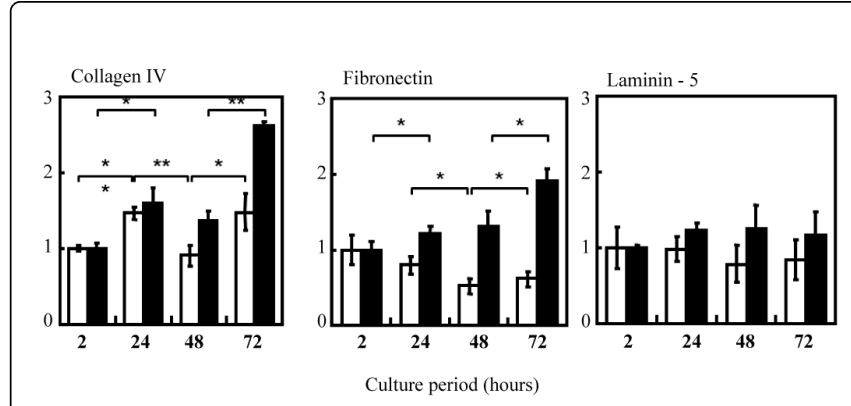

Figure 7: Culture time-dependence of relative expression levels of m-RNAs encoding endothelium-specific markers such as collagen IV (upper), laminin 5 (middle), and FN (lower) expressed on VEGF- and FN-bound substrates. Data are expressed as means \pm standard deviation for 3 independent total RNA samples. ${ }^{*} \mathrm{p}<0.05$, ${ }^{* *} \mathrm{p}<0.01$

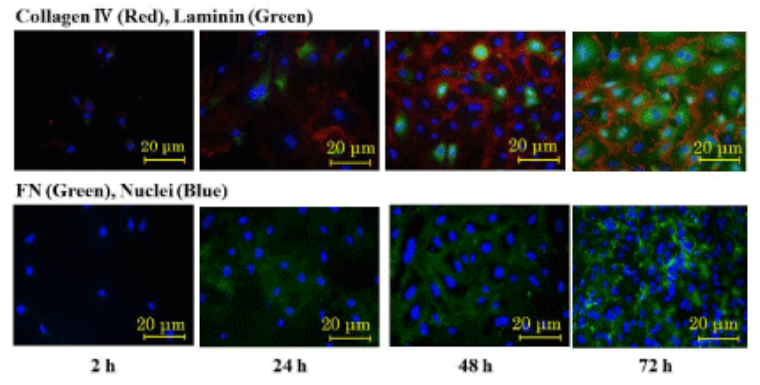

Figure 8: Representative immunofluorescence images of cells cultured for up to 72 hours on VEGF-bound substrate. Collagen IV and laminin are represented by red and green, respectively (upper). FN and nuclei are represented by green and blue, respectively (lower). Deposition of collagen IV, FN, and laminin increased with time. Collagen IV and FN appear to localize at the cell-cell interfaces or in the peripheral regions of cells, whereas laminin appears to distribute homogeneously beneath the cells. Scales are 20 micrometers

\section{Discussion}

Due to the promising therapeutic benefits of EPCs, several approaches have recently been developed to capture EPCs directly from the blood stream [8]. The main theme of EPC-capture technology is exploration of active biomolecules that target cell-surface molecules specifically expressed on EPCs. In addition to anti-CD34 antibody-bound stents, synthetic biomolecules used for this purpose have included a single-stranded DNA aptamer against CD31 (also called PECAM-1), cyclic RGD (Arg-Gly-Asp, a common motif that binds to integrins) targeting EPC-specific integrins and peptides obtained from phage-display screens that specifically recognize EPCs [11,12,22-24]. Although these surface-bound biomolecules exhibit selective adhesion of EPCs in vitro, animal studies are still inconclusive.

Recent studies showed that, in order to avoid adhesion of unfavorable blood cells described above, selective capture of EPCs 
followed by endothelialization and minimized intimal hyperplasia could be achieved by anti-VE-cadherin-bound stents in a rabbit model. Using a conceptually similar approach, we have independently explored in situ capture technology using a different cell-surface molecule exclusively expressed on endothelial-lineage cells $[25,26]$. EPCs are a type of pre-differentiated adult stem cells with high proliferation and differentiation potentials. During culture, these cells undergo time-dependent alterations in phenotype. Therefore, in this study we used ECs, which are terminally differentiated cells.

Among various interacting cell-surface markers and ligands, schematically shown in Figure 1, only surface-bound VEGF exhibited adhesion, spreading, and proliferation potentials and these properties were comparable to those of surface-bound FN. This is in good agreement with the results of our previous studies using mononuclear cells on VEGF-bound surfaces, in which we observed high adhesion, proliferation, and differentiation potentials, as well as high adhesive strength against simulated arterial shear stress $[17,18]$.

On the other hand, it has been reported that, after 30 minutes of adhesion of ECs and EPCs, surface-bound VEGF conjugated with heparin phosphorylates exogenous VEGFR-2; and at the same time there is an increase in PECAM-1 expression, which is a consequence of VEGF stimulation [27]. However, the time-dependent phosphorylation profile has not been studied previously. In this study, interaction of surface-bound VEGF with VEGFR eventually led to prolonged activation of intracellular signal-transduction pathways. Phosphorylation of VEGFR, persisting about one day, was observed in the early culture period, but little phosphorylation was observed subsequently (Figure 6A). In the case of FAK, which acts downstream of VEGF signaling, phosphorylation was up-regulated in the early culture period but was down-regulated later, synchronous with the phosphorylation of VEGFR (Figure 6B). Soluble VEGF has been reported to activate the FAK signal pathway through VEGFR, resulting in reorganization of cytoskeletal architecture due to actin-filament assembly. Phosphorylation of the ERK and Akt continued for a few days after adhesion (Figures 7A and 7B), which may contribute to proliferation, survival and differentiation processes, although whether these signals are transmitted directly from phosphorylated VEGFR or indirectly through phosphorylated FAK remains unclear.

The prolonged phosphorylation of these intracellular signaling molecules triggered by surface-bound VEGF is in marked contrast with the phosphorylation profiles of soluble VEGF added into the culture medium; very short transient activation (on the order of minutes) is commonly observed for soluble growth factors, due to spontaneous internalization followed by de-phosphorylation. These results may indicate that surface-bound VEGF actively participates in adhesion, spreading, and proliferation, which proceed even after a prolonged culture period. It is intriguing to determine how long the VEGF/VEGFR adhesion mechanism lasts. As shown in Figure 7, the levels of mRNAs encoding collagen IV and FN on VEGF-bound substrate increased with culture time. Concomitantly, synthesis and secretion of these macromolecules into the cytoplasm, as determined by immunofluorescence staining, increased with culture time (Figure 8).

Although precise analysis by confocal laser-scanning microscopy is necessary, the evidence presented so far suggests that VEGF/VEGFR adhesion on a "provisional" cell-adhesion platform (solid-phase VEGF) diminishes with time in early culture periods; within a few days after adhesion, however, adhesion transforms to an integrindriven mechanism on newly deposited, naturally occurring ECM molecules. Once such an adhesion mechanism predominates, a firm and integrated endothelium may be created. In addition, our previous study reported that simulated arterial shear stress augments activation of intracellular signal-transduction pathways and differentiation processes of EPCs that have adhered to VEGF-bound stents, which should provide beneficial effect on endothelialization process in the bloodstream. In fact, our extensive study of stenting with VEGFbound stents in porcine coronary model showed faster capture of EPCs, higher degree of endothelium formation, and much thinner intimal layer as compared with those of anti-CD34 antibody-bound stents, although further improvement of polymer should be considered $[19,28,29]$.

\section{Conclusion}

A VEGF-bound surface can serve as a platform for highly selective EPC capture by bio-specific ligand binding between cell membrane VEGFR and surface-bound VEGF. This also brings about accelerated differentiation and endothelialization by prolonged stimulation of the associated signaling pathway by solid-phase VEGF and by hydrodynamic shear stress in arterial bloodstream. These results may deepen understanding of cellular events and the fate of in- situ captured EPCs and provide a logical basis for use of a VEGF-bound surface platform as a preferred platform of bioactive stents.

\section{Acknowledgements}

This study was financially supported by government funds: the Toyama/Ishikawa Regional Invention Project (2008-2012), Grants-inAid for Scientific Research B (2006-2008) (both to T.M.), and the High-Tech Research Project (2004-2013), all of which are from the Ministry of Education, Culture, Sports, Science and Technology of Japan. The authors appreciate technical advice and assistance provided by Dr. M. Kogi, T. Shinmura, C. Ariga, and M. Hashida.

\section{References}

1. Xue L, Greisler HP (2003) Biomaterials in the development and future of vascular grafts. J Vasc Surg 37: 472-480.

2. Matsuda T (2004) Recent progress of vascular graft engineering in Japan. Artif Organs 28: 64-71.

3. Deutsch M, Meinhart J, Fischlein T, Preiss P, Zilla P (1999) Clinical autologous in vitro endothelialization of infrainguinal e PTFE grafts in 100 patients: a 9-year experience. Surgery 126: 847-855.

4. Asahara T, Murohara T, Sullivan A, Silver M, van der Zee R, et al. (1997) Isolation of putative progenitor endothelial cells for angiogenesis. Science 275: 964-967.

5. Kaushal S, Amiel GE, Guleserian KJ, Shapira OM, Perry T, et al. (2001) Functional small-diameter neo-vessels created using endothelial progenitor cells expanded ex vivo. Nat Med 7: 1035-1040.

6. He H, Shirota $T$, Yasui H, Matsuda $T$ (2003) Canine endothelial progenitor cell-lined hybrid vascular graft with non-thrombogenic potential. J Thorac Cardiovasc Surg 126: 455-464.

7. Shirota $\mathrm{T}$, He H, Yasui H, Matsuda $\mathrm{T}$ (2003) Human endothelial progenitor cell-seeded hybrid graft: proliferative and anti-thrombogenic potentials in vitro and fabrication processing. Tissue Eng 9: 127-136.

8. Liu W, Peng Y, Wu B, Li Q, Chai H, et al. (2013) A meta-analysis of the impact of EPC capture stent on the clinical outcomes in patients with coronary artery disease. J Interv Cardiol 26: 228-238.

9. Avci-Adali M, Paul A, Ziemer G, Wendel HP (2008) New strategies for in vivo tissue engineering by mimicry of homing factors for selfendothelialisation of blood contacting materials. Biomaterials 29: 3936-3945. 
Citation: Tagawa S, Matsuda T, Aomizu T, Kuwana M, Ohtake H, et al. (2014) Surface-Bound Vascular Endothelial Growth Factor Promotes Prolonged Activation of Endothelial Cells: A New Technology for Capturing Endothelial Progenitor Cells by Intravascular Stents. J Tissue Sci Eng 5: 1000140. doi:10.4172/2157-7552.1000140

Page 7 of 7

10. Aoki J, Serruys PW, van Beusekom H, Ong AT, McFadden EP, et al. (2005) Endothelial progenitor cell capture by stents coated with antibody against CD34: the HEALING-FIM (Healthy Endothelial Accelerated Lining Inhibits Neo-intimal Growth-First In Man) Registry. J Am Coll Cardiol 45: 1574-1579.

11. Co M1, Tay E, Lee CH, Poh KK, Low A, et al. (2008) Use of endothelial progenitor cell capture stent (Genous Bio-Engineered R Stent) during primary percutaneous coronary intervention in acute myocardial infarction: intermediate- to long-term clinical follow-up. Am Heart J 155: 128-132.

12. Rotmans J, Heyligers JM, Verhagen HJ, Velema E, Nagtegaal MM, et al. (2005) In vivo cell seeding with anti-CD34 antibodies successfully accelerates endothelialization but stimulates intimal hyperplasia in porcine arteriovenous expanded polytetrafluoroethylene grafts. Circulation 112: 12-18.

13. Lim WH, Seo WW, Choe W, Kang CK, Park J, et al. (2011) Stent coated with antibody against vascular endothelial-cadherin captures endothelial progenitor cells, accelerates re-endothelialization, and reduces neointimal formation. Arterioscler Thromb Vasc Biol 31: 2798-2805.

14. Tan A, Goh D, Farhatnia Y, G Natasha, Lim J, et al. (2013) An anti-CD34 antibody-functionalized clinical-grade POSS-PCU nano-composite polymer for cardiovascular stent coating applications: a preliminary assessment of endothelial progenitor cell capture and hemocompatibility. PLoS One 8:e77112. doi: 10.1371/journal.pone.0077112. e Collection 2013.

15. Ma X, Hibbert B, McNulty M, Hu T, Zhao X, et al. (2014) Heat shock protein 27 attenuates neo-intima formation and accelerates reendothelialization after arterial injury and stent implantation: importance of vascular endothelial growth factor up-regulation. FASEB J 28: 594-602.

16. Miyazu K, Kawahara D, Ohtake H, Watanabe G, Matsuda T (2010) Luminal surface design of electrospun small-diameter graft aiming at in situ capture of endothelial progenitor cell. J Biomed Mater Res B Appl Biomater 94: 53-63.

17. Kawahara D1, Matsuda T (2012) Hydrodynamic shear-stress-dependent retention of endothelial and endothelial progenitor cells adhered to vascular endothelial growth factor-fixed surfaces. J Biomed Mater Res B Appl Biomater 100: 1218-1228.

18. Matsuda T, Kuwana M, Aomizu T, Yamagishi M, Ohtake H, et al. (2013) Surface design for in situ capture of endothelial progenitor cells: VEGFbound surface architecture and behaviors of cultured mononuclear cells. J Biomed Mater Res B Appl Biomater 101: 50-60.
19. Takabatake S, Hayashi K, Nakanishi C (2014) Vascular endothelial growth factor-bound stents: application of in-situ capture technology of circulating endothelial progenitor cells in porcine coronary model. J Interv Cardiol 27: 63-72.

20. Zachary I (2003) VEGF signalling: integration and multi-tasking in endothelial cell biology. Biochem Soc Trans 31: 1171-1177.

21. Favata MF, Horiuchi KY, Manos EJ, Daulerio AJ, Stradley DA, et al. (1998) Identification of a novel inhibitor of mitogen-activated protein kinase kinase. J Biol Chem 273: 18623-18632.

22. Hoffmann J, Paul A, Harwardt M, Groll J, Reeswinkel T, et al. (2008) Immobilized DNA aptamers used as potent attractors for porcine endothelial precursor cells. J Biomed Mater Res A 84: 614-621.

23. Thébaud NB, Pierron D, Bareille R, Le Visage C, Letourneur D, et al. (2007) Human endothelial progenitor cell attachment to polysaccharidebased hydrogels: a pre-requisite for vascular tissue engineering. J Mater Sci Mater Med 18: 339-345.

24. Veleva AN, Cooper SL, Patterson C (2007) Selection and initial characterization of novel peptide ligands that bind specifically to human blood outgrowth endothelial cells. Biotechnol Bioeng 98: 306-312.

25. Lim WH, Seo WW, Choe W, Kang CK, Park J, et al. (2011) Stent coated with antibody against vascular endothelial-cadherin captures endothelial progenitor cells, accelerates re-endothelialization, and reduces neointimal formation. Arterioscler Thromb Vasc Biol 31: 2798-2805.

26. Lee JM, Choe W, Kim BK, Seo WW, Lim WH, et al. (2012) Comparison of endothelialization and neo-intimal formation with stents coated with antibodies against CD34 and vascular endothelial-cadherin. Biomaterials 33: 8917-8927.

27. Anderson SM, Chen TT, Iruela-Arispe ML, Segura T (2009) The phosphorylation of vascular endothelial growth factor receptor-2 (VEGFR-2) by engineered surfaces with electrostatically or covalently immobilized VEGF. Biomaterials 30: 4618-4628.

28. Suzuki Y, Yamamoto K, Ando J, Matsumoto K, Matsuda T (2012) Arterial shear stress augments the differentiation of endothelial progenitor cells adhered to VEGF-bound surfaces. Biochem Biophys Res Commun 423: 91-97.

29. Tan A, Farhatnia Y, Goh D, G Natasha, de Mel A, et al. (2013) Surface modification of a polyhedral oligomeric silsesquioxane poly(carbonateurea) urethane (POSS-PCU) nano-composite polymer as a stent coating for enhanced capture of endothelial progenitor cells. Bio interphases 8: 22. 